DNA ORIGAMI

\section{Slider and hinge joints}

Proc. Natl Acad. Sci. USA http://doi.org/x4p (2015)
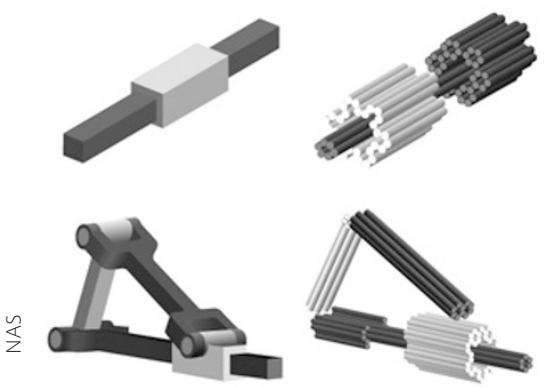

Mechanical machines combine joints and hinges to perform complex motion patterns. DNA machines could in principle perform similar three-dimensional motion, yet the design and assembly of DNA origami structures that undergo reversible conformational changes is at a comparatively primitive stage. Carlos Castro and colleagues now show that mechanical devices capable of precise constrained linear motion at the nanoscale, which has so far been difficult to achieve, can be designed and fabricated by following macroscopic-machine design. By combining stiff double-stranded DNA origami components and flexible singlestranded DNA connections, the researchers made crank-sliders (which couple linear and rotational motion) and combinations of hinges and links that make up mechanical linkages. They also show that by employing DNA strand displacement (typically used to reconfigure DNA origami nanostructures) the linkages can move within minutes between open-frame and compact-bundle configurations. The modular design of kinematic joints made of DNA origami should facilitate the design of programmable complex DNA nanomachines for use in, for example, biosensing or triggered actuation.

\section{SPIN TRANSISTORS}

\section{Going electric}

Nature Nanotech. 10, 35-39 (2015)

Many efforts have been devoted to the development of spin field-effect transistors since they were proposed by Datta and Das in 1990. Their idea was to inject and detect spin-polarized currents in a two-dimensional gas of a semiconductor heterostructure by using magnetic electrodes, and to control the spin polarization with electric fields, which would allow the encoding of information. The fabrication of the device is challenging however due to reduced spin-injection efficiencies and spin-depolarization effects. Optical pumping and magnetic field modulations through the transport channel have been shown to be efficient approaches to overcome these issues, but pose additional difficulties for their largescale integration in semiconductor-based circuits. Now, Pojen Chuang and colleagues use two quantum point contacts produced by applying voltages to split gates patterned on the semiconductors as spin injectors and detectors. This solves earlier integration issues and allows spin-injection efficiencies close to $100 \%$. DC

\section{AMYLOID SELF-ASSEMBLY}

\section{Dimer detection}

Angew. Chem. Int. Ed. http://doi.org/ł2xrdh (2014)

The presence of amyloid fibrils is characteristic of several diseases, including Alzheimer disease and type 2 diabetes. Hence, the formation of the peptide dimers and oligomers, which subsequently aggregate to make these fibrils, is an important process to monitor and understand. Now, Ehud Gazit and colleagues report an assay with the ability to detect dimerization of three amyloidogenic polypeptide monomers; human islet amyloid polypeptide, $\beta$-amyloid or $\alpha$-synuclein. The bimolecular fluorescence complementation

\section{FLEXIBLE ELECTRONICS}

\section{All-organic image detectors}

Adv. Mater. http://doi.org/f2xrf2 (2014)

Photodetectors based on organic materials are appealing alternatives to silicon devices because of their low cost, flexibility and sensitivity. In an efficient organic two-dimensional array working as an image detector, each pixel of the array is composed of a light-sensing element, a memory unit that stores the detected information and a selector that minimizes crosstalk - interference from adjacent pixels on the electric signal read from a specific unit. Sebastian Nau and colleagues now show that compact photosensor-storage-selector systems integrated in highly dense flexible arrays are obtained by vertically connecting in series an organic photodiode and an organic resistive switch. When exposed to light, the photodiode decreases its resistivity and the voltage applied to the series circuit drops across the resistive switch changing its status from high- to low-resistance. This status is preserved until a signal is applied to reset the switch. The nonlinear behaviour of the photodiode is used to minimize crosstalk during read-out. assay relies on fluorescence signals only resulting from dimeric interaction between the amyloidogenic peptides, which are fused, via a linker, to fragments of a split mCherry protein. The assay identified compounds already known to inhibit aggregation of amyloid peptides and these compounds were then shown to increase the viability of pancreatic cells in the presence of amyloid fibrils in a dose-dependent manner. The correlation between the screening process and in vitro results indicates that these dimeric interactions are an important target for drug development. The assay has the potential to be automated, thus permitting high-throughput screening of compounds. AS

\section{GRAPHENE}

\section{Supersonic impact}

Science 346, 1092-1096 (2014)

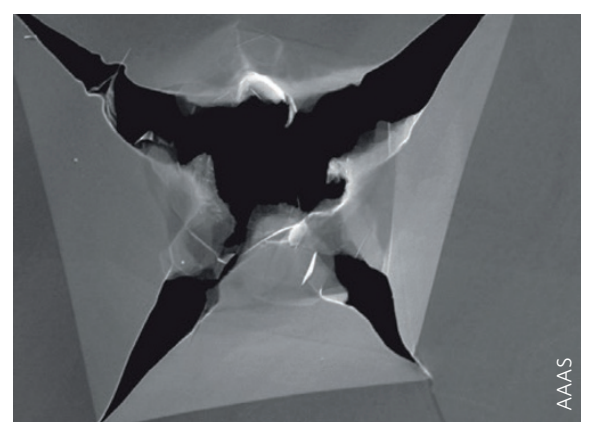

Nano- and micromechanical testing of graphene have previously revealed a number of attractive properties, including exceptional strength and stiffness, attributable to the covalent bonding between carbon atoms in the single-atom layer. So far its mechanical properties have been mainly investigated under quasistatic loading; response to dynamic loads is relatively unknown. Jae-Hwang Lee and colleagues now explore this regime by performing impact testing by firing single micrometre-sized silica spheres at supersonic speeds at multilayer graphene, achieving strain rates of $\sim 10^{7} \mathrm{~s}^{-1}$. They report that the penetration energy can be 8-12 times those of literature values for steel, at equivalent projectile velocities. This result can be explained in part by a large in-plane speed of sound - resulting from graphene's low density and high stiffness - that permits rapid delocalization of concentrated stresses during projectile impact, mitigating premature failure. Such high resistance to failure under dynamic loading, coupled with it now being possible to synthesize large-area sheets, might make graphene an option in composite armour systems.

Written by David Ciudad, Luigi Martiradonna, Pep Pàmies, John Plummer and Alison Stoddart. 